\title{
Prospective study of the value of transbronchial lung biopsy after lung transplantation
}

\author{
A. Boehler, P. Vogt, A. Zollinger, W. Weder, R. Speich
}

\begin{abstract}
Prospective study of the value of transbronchial lung biopsy after lung transplantation. A. Boehler, P. Vogt, A. Zollinger, W. Weder, R. Speich. (CERS Journals Ltd 1996.

ABSTRACT: Transbronchial lung biopsy (TBB) has become the gold standard for the diagnosis of acute rejection and cytomegalovirus (CMV) pneumonia in lung transplant recipients. The aim of this study was to assess the value of regular surveillance TBB in stable asymptomatic patients and to establish the role of TBB as a follow-up procedure 1 month after a previous pathological biopsy result.

We prospectively evaluated $76 \mathrm{TBBs}$ performed in 17 lung transplant recipients.

A definite pathological result was found in 14 of 15 TBBs performed for clinical indications: CMV pneumonia (5), acute rejection grade $\geq A 2$ according to the criteria of the International Society for Heart and Lung Transplantation (ISHLT) (4), bronchiolitis obliterans (3), and desquamative interstitial pneumonitis (2). Fifteen of 45 surveillance TBBs performed in asymptomatic patients revealed significant abnormalities. Ten episodes of acute rejection ISHLT grade $\geq \mathbf{A} 2$ and three episodes of CMV pneumonia detected by TBB had direct therapeutic consequences. Nine of 16 follow-up TBBs performed 1 month after a pathological biopsy result again showed relevant pathological findings. With the exception of one severe haemorrhage, no life-threatening complications occurred.

Our results suggest that transbronchial lung biopsies performed on a regular basis after lung transplantation are important for the detection of asymptomatic and/or persistent acute rejection or infection. In the long-term, this strategy might be the most effective tool in reducing the incidence of bronchiolitis obliterans, which is still the main obstacle for further improvement of long-term survival after lung transplantation.
\end{abstract}

Eur Respir J., 1996, 9, 658-662.
Depts of Internal Medicine, Pathology, Anesthesiology and Surgery, University Hospital Zurich, Zurich, Switzerland.

\author{
Correspondence: R. Speich \\ Dept of Internal Medicine \\ University Hospital Zurich \\ Raemistr. 100 \\ CH-8091 Zurich \\ Switzerland
}

Keywords: Bronchiolitis obliterans lung transplantation rejection

transbronchial biopsy

Received: August 221995

Accepted after revision January 81996
During the last decade, lung transplantation has evolved into a successful therapeutic modality for endstage diseases of the lungs and the pulmonary circulation [1-6]. According to the St. Louis International Lung Transplant Registry, 1, 2 and 3 year survival rates of 71, 64 and $58 \%$, respectively, can be achieved (St. Louis International Lung Transplant Registry, April 1995 Report). This success is mainly due to cautious selection of patients, improved surgical techniques and organ preservation, as well as careful postoperative management. The major complications in survivors of the perioperative period of lung transplantation are infections and episodes of acute rejection. However, the main obstacle for long-term success of lung transplantation remains chronic rejection [7, 8]. Chronic rejection occurs in up to two thirds of patients who have survived the first year after surgery [9-11]. Generally accepted clinical features of chronic rejection include a progressive fall in forced expiratory volume in one second (FEV1) along with increasing dyspnoea on exertion [12].

Chronic lung rejection is characterized histologically by bronchiolitis obliterans, with a variable degree of pulmonary vascular involvement [7, 8]. Recently, the term "bronchiolitis obliterans syndrome" (BOS) has been adopted to describe allograft deterioration secondary to progressive airway disease for which no other cause is detectable [12]. Whilst the term "bronchiolitis obliterans" is reserved for histologically proven diagnosis only, the diagnosis of "bronchiolitis obliterans syndrome" does not necessarily require histological confirmation [12]. The value of enhanced immunosuppressive therapy in patients with BOS remains unknown up to now, and at least one third of the patients will progress to severe respiratory failure and eventually die, retransplantation being the only effective management. The most important factor for the development of BOS is the number of previous acute rejection episodes and the incidence of persistent rejection after treatment of acute rejection episodes $[9,10]$.

Signs and symptoms as well as radiographic features and bronchoalveolar lavage findings are nonspecific and, therefore, not helpful in differentiating between acute rejection and other processes, such as infectious complications. Higenbottam et al. [13] have established the role of transbronchial lung biopsy (TBB) as the "gold standard" for the diagnosis of acute rejection after lung transplantation. Recently, TRULOCK et al. [14] have shown that in TBBs performed for surveillance in clinically and physiologically stable recipients, the incidence of acute rejection and of cytomegalovirus (CMV) pneumonia was 
unexpectedly high. Almost $40 \%$ of surveillance biopsies disclosed acute rejection.

Therefore, when we started our lung transplant programme, we decided to perform regular surveillance biopsies in our patients. We report the results of our first 76 transbronchial lung biopsies, especially with respect to their role in the surveillance of patients after lung transplantation.

\section{Methods}

\section{Patients}

Between November 1992 and September 1994, 20 lung transplants were performed in 19 recipients [15]. One patient underwent retransplantation for severe bronchiolitis obliterans 624 days after the first operation. Fourteen patients received a single lung transplant, and six a bilateral lung transplant. Sixteen patients are alive after a median survival time of 409 days (range 117-789 days). Four patients died 11, 76, 203 and 230 days after lung transplantation, due to multiple-organ failure, CMV pneumonia with subsequent systemic fungal infection, severe BOS, and encephalitis of unknown aetiology, respectively. All transplants were ABO-matched, and none was human leucocyte antigen (HLA)-matched. In eight instances, the CMV serology both of recipient and donor was negative. In seven cases both recipient and donor were CMV seropositive. Five patients were seronegative for CMV and received a lung transplant from a CMV seropositive donor.

The induction immunosuppressive regimen consisted of cyclosporin, azathioprine, and antilymphocyte globulin, which was administered for 5-7 days. Maintenance immunosuppressive drugs were cyclosporin (monoclonal cyclosporin A trough level $180-250 \mu \mathrm{g} \cdot \mathrm{L}^{-1}$ ), azathioprine $\left(2 \mathrm{mg} \cdot \mathrm{kg}^{-1}\right.$ q.d.), and prednisone $\left(0.5 \mathrm{mg} \cdot \mathrm{kg}^{-1}\right.$ q.d., tapered to $10-15 \mathrm{mg}$ q.d. within the first six postoperative months). Acute rejection episodes were treated with prednisone pulses (0.5-1 g q.d.) for 3 days. Antilymphocyte globulin was given in three patients, and OKT 3 in one patient with recurrent acute rejection and/or bronchiolitis obliterans.

\section{Transbronchial lung biopsy}

Bronchoscopy was performed under topical anaesthesia and intravenous sedation. The patients received 0.5 $\mathrm{mg}$ atropine sulphate and 7.5-15 mg hydrocodone subcutaneously $15 \mathrm{~min}$ before fibreoptic bronchoscopy. Intravenously administered flunitrazepam was used for additional sedation. The patients received $5 \mathrm{~mL}$ nebulized $4 \%$ lidocaine followed by $10 \mathrm{~mL}$ of $1 \%$ lidocaine injected through the fibreoptic bronchoscope onto the vocal cords. Prior to biopsy, bronchoalveolar lavage was performed with 3-4 $50 \mathrm{~mL}$ aliquots of $0.9 \% \mathrm{NaCl}$ instilled and gently aspirated from a subsegmental bronchus of either the lingula or the middle lobe. Transbronchial lung biopsies were obtained from the lung periphery under fluoroscopic guidance with a $2 \mathrm{~mm}$ fenestrated forceps (Olympus Optical AG, Volketswil, Switzerland). Three to five biopsies were obtained from each lobe of one lung. The biopsy specimens were fixed in $4 \%$ formaldehyde solution, and then serially sectioned and stained with haematoxylin and eosin, elastic van Gieson and Grocott.

The histological diagnosis of acute rejection was based on the presence of a perivascular mononuclear infiltrate. The severity of acute rejection was graded according to the criteria of the International Society for Heart and Lung Transplantation (ISHLT) [16]: grade A0 = no significant abnormality; grade A1 = minimal; grade A2 = mild; grade $\mathrm{A} 3$ = moderate; and grade $\mathrm{A} 4=$ severe . Further aspects of pulmonary rejection were also graded according to the working formulation of ISHLT: grade $\mathrm{B}=$ active airway damage without scarring; grade B1 = lymphocytic bronchitis; grade B2 = lymphocytic bronchiolitis; grade $\mathrm{C}=$ chronic airway rejection; grade $\mathrm{C} 1$ = subtotal bronchiolitis obliterans; grade $\mathrm{C} 2=$ total bronchiolitis obliterans; grade $\mathrm{D}=$ chronic vascular rejection; grade $\mathrm{E}$ = vasculitis. CMV pneumonia was identified histologically by characteristic intracellular inclusions and concurrent inflammatory changes. The presence of the virus was confirmed by in situ hybridization with commercially available probes (Enzo Diagnostics). Aspergillus pneumonia was confirmed by typical branching hyphae invading tissue.

\section{Indications for biopsy}

The indication for each procedure was prospectively categorized as "clinical", "surveillance" or "follow-up". "Clinical" procedures were performed in patients with new symptoms (i.e. dyspnoea, cough), signs (i.e. fever, crackles), roentgenographic infiltrates, or declining lung function (i.e. decrease of FEV1 of $\geq 10 \%$ from the baseline). "Surveillance" procedures were carried out in clinically and physiologically stable recipients monthly during the first 6 postoperative months, and 3-6 monthly thereafter. "Follow-up" procedures were routinely performed 4 weeks after a previous biopsy with a significant pathological finding.

Bronchiolitis obliterans syndrome (BOS) was defined according to the working formulation of the ISHLT [12]: grade $0=$ no significant abnormality, i.e. FEV $180 \%$ or more of baseline value after transplantation; grade $1=$ mild BOS, i.e. FEV1 $66-80 \%$ of the baseline value; grade $2=$ moderate $\mathrm{BOS}$, i.e. FEV $151-65 \%$ of the baseline value; grade $3=$ severe $\mathrm{BOS}$, i.e. $\mathrm{FEV} 1 \leq 50 \%$ of baseline value. Each grade is subdivided in "a" (without pathological evidence of bronchiolitis obliterans) and "b" (with pathological evidence of bronchiolitis obliterans).

\section{Data analysis}

Statistical comparison between groups were performed with Mann-Whitney U-test for continuous or Fisher's exact test for discrete variables.

\section{Results}

A total of 76 transbronchial lung biopsies were performed in 17 of 20 lung transplants. In three patients 
no biopsy could be obtained; due to early fatal multipleorgan failure in one patient, and a complicated postoperative course with prolonged mechanical ventilation in two. The average number of procedures per recipient was 4.1 (range 1-9). Thirteen TBBs were obtained for clinical indications, 45 biopsies were performed for surveillance, and 16 procedures were performed for followup. All procedures revealed adequate histological specimens, i.e. a minimum of five TBBs containing pulmonary parenchyma with at least 50-100 alveoli. The results of the 76 TBBs are summarized in table 1 .

Thirty eight biopsies disclosed no significant pathological changes, i.e. either acute rejection ISHLT grade $\leq \mathrm{A} 1$ or absence of infection. Specific histological features of acute rejection or infection were found in 36 TBBs. Additionally, one patient exhibited desquamative interstitial pneumonitis in two TBBs, possibly due to recurrence of the underlying lung disease (idiopathic pulmonary fibrosis). Although the patient improved after augmented immunosuppression, this histological finding may be considered as nonspecific. Acute rejection ISHLT grade $\geq \mathrm{A} 2$ was found in 17 biopsies ( 8 patients), CMV pneumonia in 12 biopsies ( 6 patients), bronchiolitis obliterans (ISHLT grade C1 or C2) in 4 biopsies (3 patients), lymphocytic bronchitis (ISHLT grade B1) in 2 biopsies (2 patients), and invasive aspergillosis in one biopsy. Three biopsies with CMV pneumonia additionally disclosed features of acute rejection ISHLT grade A2.

Overall, a relevant pathological biopsy result was found in 14 of 15 clinical procedures, in 15 of 45 surveillance procedures, and in 9 of 16 follow-up procedures (table 1).

The TBBs performed for clinical indications revealed: CMV pneumonia in five (concurrent perivascular lymphocytic infiltrate ISHLT grade A2 in one biopsy); acute rejection ISHLT grade $\geq \mathrm{A} 2$ in four; bronchiolitis obliterans (ISHLT C1 or C2) in three; and desquamative interstitial pneumonitis in two procedures. Only one biopsy was normal. This patient suffered from severe bacterial bronchitis (Pseudomonas aeruginosa) without infiltrates on chest radiograph and recovered completely after antibiotic treatment. Thus, the sensitivity for relevant pathological parenchymatous changes of TBBs performed as a clinical procedure was $100 \%$ (14 out of 14).

The 45 TBBs performed for surveillance in asymptomatic and functionally stable patients revealed pathological abnormalities in 15 procedures. Thirteen biopsy results had important clinical implications, such as antirejection treatment in 10 , and commencement of intravenous ganciclovir therapy in three cases. In two patients, the biopsies revealed lymphocytic bronchitis (ISHLT grade B1) which, however, was not treated by augmented immunosuppression.

In 9 of 16 TBBs performed for follow-up 1 month after a previous pathological biopsy result, the following relevant histological abnormalities could be detected: persistent acute rejection episodes ISHLT grade A2 or greater in three; nonresolved CMV pneumonia in four; and bronchiolitis obliterans (ISHLT grade C1) and invasive aspergillosis after augmented immunosuppression in one patient each.

No lethal complications occurred due to TBB. One patient developed severe haemorrhage (about $300 \mathrm{~mL}$ ) requiring intubation and mechanical ventilation for $6 \mathrm{~h}$. The haemorrhage could be managed by tamponade with the fibreoptic bronchoscope and intravenous application of ornipressin. Five patients had moderate haemorrhages with an estimated blood loss of 100-200 mL, subsequently controlled by tamponade wedging with the fibreoptic bronchoscope. Three episodes of pneumothorax requiring chest tube drainage occurred in a single patient, who probably had an anomaly of the right middle lobe bronchus (on computed tomography a large bronchus extended to the anterior pleura). Hence, the overall complication rate was $12 \%$ (9 out of 76 ).

Four patients ( 1 male and 3 females) developed bronchiolitis obliterans. In three cases, a histological diagnosis was made by transbronchial biopsy and in one case at autopsy. Two patients suffered from BOS grade $3 \mathrm{~b}$ (one patient was retransplanted 624 days after the first transplantation, and one patient died 203 days after transplantation due to respiratory failure). Two patients suffered from stable mild BOS (grade 1b), which was diagnosed 86 and 269 days after lung transplantation, respectively. Whereas at least two episodes of acute rejection ISHLT $\geq \mathrm{A} 2$ occurred in 2 of 16 patients without BOS, two of the patients with BOS had 3 and 5 acute rejection episodes of ISHLT grade $\geq \mathrm{A} 2$, respectively $(\mathrm{p}=0.16)$. The remaining two patients underwent biopsies only twice, since BOS had already developed within 3 months after transplantation, and one acute rejection episode of ISHLT grade A2 was detected in each case.

Overall, the four patients with subsequent BOS suffered from a total of 10 acute rejection episodes of ISHLT grade $\geq \mathrm{A} 2$ in 21 biopsy procedures, whereas only seven such events were found in $55 \mathrm{TBBs}$ in the cases without $\operatorname{BOS}(\mathrm{p}=0.016)$.

Table 1. - Results of 76 transbronchial lung biopsies in 17 lung transplant recipients

\begin{tabular}{lccc}
\hline & $\begin{array}{c}\text { Clinical } \\
(\mathrm{n}=15)\end{array}$ & $\begin{array}{c}\text { Surveillance } \\
(\mathrm{n}=45)\end{array}$ & $\begin{array}{c}\text { Follow-up } \\
(\mathrm{n}=16)\end{array}$ \\
\hline No significant pathology (i.e. rejection ISHLT grade $\leq \mathrm{A} 1$ or lack of infection) & 1 & 30 & 7 \\
Acute rejection ISHLT grade $\geq$ A2 & 4 & 10 & 3 \\
CMV pneumonia (with concurrent signs of acute rejection $\geq \mathrm{A} 2)$ & $5(1)$ & $3(2)$ & 4 \\
Bronchiolitis obliterans (ISHLT grade C1 or C2) & 3 & - & 1 \\
Lymphocytic bronchitis (ISHLT grade B1) & - & 2 & - \\
Desquamative interstitial pneumonitis & 2 & - & - \\
Invasive aspergillosis & - & - & 1 \\
\hline Total of biopsies with significant pathology & 14 & 15 & 9 \\
& $(93 \%)$ & $(33 \%)$ & $(56 \%)$ \\
\hline
\end{tabular}

ISHLT: International Society for Heart and Lung Transplantation; grade A1: minimal abnormality; grade A2: mild abnormality; grades $\mathrm{C} 1$ and $\mathrm{C} 2$ : subtotal and total bronchiolitis obliterans, respectively; grade $\mathrm{B} 1$ : lymphocytic bronchiolitis; CMV: cytomegalovirus. 


\section{Discussion}

The results of our prospective study emphasize the importance of transbronchial lung biopsies for the postoperative management of lung transplant recipients. In 93\% of the biopsies performed for clinical indications, relevant pathological changes could be detected. The only patient with deteriorating lung function but negative biopsy results suffered from severe bacterial bronchitis and improved after antimicrobial therapy. Thus, the high diagnostic sensitivity (90-100\%) for transbronchial lung biopsy in lung transplant recipients with deteriorating graft function described by others $[13,14,17]$ was confirmed by our findings. In this setting, transbronchial lung biopsy serves to differentiate between acute rejection and infection, since clinical symptoms and signs as well as radiological and laboratory findings are nonspecific and, therefore, nondiagnostic [13]. Furthermore, as in one of our patients, cases with coincident rejection and infection may be detected $[14,18]$. The significance of a perivascular lymphocytic infiltrate in patients with infections such as CMV pneumonia, however, is not known $[14,18,19]$. In three of our cases, transbronchial lung biopsy revealed bronchiolitis obliterans, which could subsequently be treated with early augmented immunosuppression. It is possible that the early treatment may have influenced the course of two of these patients, who stabilized in BOS grade $1 \mathrm{~b}, 4$ and 10 months after the initial diagnosis of bronchiolitis obliterans [11]. In our experience and in contrast to others [14], nonspecific findings in TBB were rare. The two biopsies with desquamative interstitial pneumonitis in one patient were interpreted as recurrence of idiopathic pulmonary fibrosis in the transplanted lung. The patient's lung function significantly improved after augmented immunosuppression. Notwithstanding, this histological finding may finally be considered as nonspecific.

The most important finding of our study is the high incidence of relevant pathological changes in the 45 surveillance biopsies. One third showed evidence of rejection and/or infection. This is in accordance with the study of De Hoyos et al. [17], who found acute rejections of ISHLT grade A2 or greater in $26 \%$ of their 30 surveillance TBBs. An even higher incidence $(57 \%)$ of significant pathological changes in their 90 surveillance TBBs was reported by TRULOCK et al. [14]. These data contrast with the earlier findings of the Papworth group [13], which in fact have established the role of TBB as the "gold standard" for the diagnosis of acute rejection after lung transplantation. In 14 routine biopsies, they found only four minimal acute rejections according to their preliminary histological grading system, and there was no rejection of clinical or therapeutic relevance [20]. The discrepancy may, in part, be explained by the diminished sensitivity of lung function testing for the early diagnosis of acute rejection after single lung transplantation. The value of lung function testing for the diagnosis of infection or rejection in transplant recipients has recently been established by the Papworth group in their heartlung transplant recipients [21]. They registered a sensitivity of 75-86\% for spirometry in detecting lung rejection or infection. In single lung transplant recipients, however, other groups have reported sensitivities of only 31$77 \%[22,23]$, depending on the underlying lung disease (higher sensitivity in single lung transplant recipients with pulmonary vascular disease). The lower sensitivity of lung function testing for detecting the evolution of pathological changes in single lung transplants may be explained by the lower volumes generated by the graft, and by the additional flow rates generated by the remaining native lung [22].

Due to the surveillance biopsies performed on a regular basis we were able to detect three cases of early CMV pneumonia and 10 acute rejection episodes of ISHLT grade $\geq A 2$. As previous acute rejection episodes are one of the most important risk factors for the development of bronchiolitis obliterans $[9,10]$, by means of regular surveillance TBBs we hope to reduce the incidence of bronchiolitis obliterans in our patient population due to earlier detection of acute rejection and subsequent treatment. This issue, however, needs to be studied further by examining more patients during a longer follow-up period. Nevertheless, in our patients the frequency of previous acute rejection episodes ISHLT grade $\geq \mathrm{A} 2$ was significantly higher in those who subsequently developed BOS compared to those without BOS.

The relevance of the two episodes of lymphocytic bronchitis (ISHLT grade B1) is unknown. According to YousEM [24], this finding may be related to previous acute rejection episodes and respond to augmented immunosuppressive therapy.

Another important finding, which is in accordance with the data of TRULOCK et al. [14], is the high incidence of persistent histological abnormalities in follow-up lung biopsies. In four patients, CMV pneumonia was shown to persist, and antiviral therapy had to be continued. In one patient, TBB revealed invasive aspergillosis which was treated with amphotericin. Furthermore, three episodes of persistent acute rejections, despite treatment of previous rejection episodes with prednisone pulses, resulted in a second course of antirejection therapy. Since persistent acute rejection episodes are also a well-known risk factor for the development of bronchiolitis obliterans [10], their early detection may have decreased the risk of subsequent bronchiolitis obliterans in our lung transplant patients.

With the exception of one episode of severe haemorrhage, which did not need surgical intervention, and three episodes of pneumothorax probably due to an anatomical variant of a middle lobe bronchus in one patient, no relevant complications occurred. Notwithstanding, considering also the five minor haemorrhages $(100-200 \mathrm{~mL})$ the overall complication rate amounted to $12 \%$, which is somewhat higher than the rate of $8.9 \%$ reported by TRulock et al. [14]. However, there may be some disparity in the definitions of haemorrhage in our study compared to theirs, because the latter only reported "excessive bleedings". An inquiry among the 17 patients (data not shown) disclosed that only one patient was apprehensive before bronchoscopy, half of the remaining patients reported feeling somewhat unpleasant and half were not at all concerned.

In conclusion, our prospective study of TBBs in lung transplant recipients demonstrates the efficacy and safety of this procedure, and confirms the high sensitivity for detecting acute rejection or infection in symptomatic patients. In this setting, TBB is the procedure of choice, and surgical lung biopsy is rarely needed. TBBs performed 
as a surveillance procedure in clinically and functionally stable lung transplant patients reveal a rather high incidence of silent acute rejection episodes or infections. Acute rejection episodes ISHLT grade $\geq \mathrm{A} 2$ occur more often in patients with subsequent BOS. We are thus convinced that TBBs performed on a regular basis, especially during the first 6 months after lung transplantation, are of great importance for the early detection of symptomatic, occult as well as persistent acute rejection episodes in these patients. Because recurrent acute and persistent acute rejection episodes are the main risk factor for the development of bronchiolitis obliterans, regular TBB procedures may help in reducing the incidence of this devastating complication of lung transplantation in the future. For confirmation of this assumption, however, further studies on more patients followed-up for a longer postoperative period are warranted.

\section{References}

1. Anonymous. Lung transplantation (Editorial). Lancet 1992; 339: 1021-1022.

2. Dark J, Corris PA. The current status of lung transplantation (Editorial). Thorax 1989; 44: 689-692.

3. De Hoyos A, Patterson GA, Maurer JR, Ramirez JC, Miller JD, Winton TL. Pulmonary transplantation: early and late results. The Toronto Lung Transplant Group. J Thorac Cardiovasc Surg 1992; 103: 295-306.

4. Higenbottam T, Otulana BA, Wallwork J. Transplantation of the lung. Eur Respir J 1990; 3: 594-605.

5. Madden B, Radley SR, Hodson M, Khaghani A, Yacoub M. Medium-term results of heart and lung transplantation. J Heart Lung Transplant 1992; 11: S241-S243.

6. Patterson GA. Lung transplantation. Semin Thorac Cardiovasc Surg 1992; 4: 71-153.

7. Maurer JR. Lung transplantation bronchiolitis obliterans. In: Epler GR, ed. Diseases of the Bronchioles. New York, Raven Press, 1994; pp. 275-289.

8. Paradis I, Yousem S, Grifith B. Airway obstruction and bronchiolitis obliterans after lung transplantation. Clin Chest Med 1993; 14: 751-763.

9. Scott JP, Higenbottam TW, Clelland CA, et al. Natural history of chronic rejection in heart-lung transplant recipients. J Heart Transplant 1990; 9: 510-515.

10. Scott JP, Higenbottam TW, Sharples L, et al. Risk factors for obliterative bronchiolitis in heart-lung transplant recipients. Transplantation 1991; 51: 813-817.

11. Theodore J, Starnes VA, Lewiston NJ. Obliterative bronchiolitis. Clin Chest Med 1990; 11: 309-321.
12. International Society for Heart and Lung Transplantation. A working formulation for the standardization of nomenclature and for clinical staging of chronic dysfunction in lung allografts. J Heart Lung Transplant 1993; 12: 713-716.

13. Higenbottam T, Stewart S, Penketh A, Wallwork J. Transbronchial biopsy for the diagnosis of rejection in heart-lung transplant patients. Transplantation 1988; 46: 532-539.

14. Trulock EP, Ettinger NA, Brunt EM, Pasque MK, Kaiser LR, Cooper JD. The role of transbronchial lung biopsy in the treatment of lung transplant recipients: an analysis of 200 consecutive procedures. Chest 1992; 102: 1049-1054.

15. Speich R, Boehler A, Zollinger A, et al. Die isolierte Lungentransplantation - Evaluation von Patienten und erste Resultate. Schweiz Med Wschr 1995; 125: 786795 .

16. Yousem SA, Berry GJ, Brunt EM, et al. A working formulation for the standardization of nomenclature in the diagnosis of heart and lung rejection: lung rejection study group. J Heart Transplant 1990; 9: 593-601.

17. De Hoyos A, Chamberlain D, Schwartzman R, et al. Prospective assessment of a standardized pathologic grading system for acute rejection in lung transplantation. Chest 1993; 103: 1813-1818.

18. Stewart S. Pathology of lung transplantation. Semin Diagn Pathol 1992; 9: 210-219.

19. Tazelaar HD. Perivascular inflammation in pulmonary infections: implications for the diagnosis of lung rejection. J Heart Lung Transplant 1991; 10: 437-441.

20. Hutter JA, Stewart S, Higenbottam T, Scott JP, Wallwork J. Histological changes in heart-lung transplant recipients during rejection episodes and at routine biopsy. $J$ Heart Transplant 1988; 7: 440-444.

21. Otulana BA, Higenbottam T, Scott J, Clelland C, Igboaka G, Wallwork J. Lung function associated with histologically diagnosed acute lung rejection and pulmonary infection in heart-lung transplant patients. Am Rev Respir Dis 1990; 142: 329-332.

22. Becker FS, Martinez FJ, Brunsting LA, Deeb MG, Flint A, Lynch JP. Limitations of spirometry in detecting rejection after single-lung transplantation. Am J Respir Crit Care Med 1994; 150: 159-166.

23. Bjortuft O, Johansen B, Boe J, Foerster A, Holter E, Geiran O. Daily home spirometry facilitates early detection of rejection in single lung transplant recipients with emphysema. Eur Respir J 1993; 6: 705-708.

24. Yousem SA. Lymphocytic bronchitis/bronchiolitis in lung allograft recipients. Am J Surg Pathol 1993; 17: 491-496. 